\title{
Comportamento de estudantes no ambiente virtual de aprendizagem de uma disciplina híbrida de produção de textos
}

\author{
Leandro S. G. Carvalho, Ana L. M. Santos, Elaine H. T. Oliveira ${ }^{1}$ \\ ${ }^{1}$ Instituto de Computação - Universidade Federal do Amazonas (UFAM) - Manaus - AM - Brasil \\ \{galvao, analuciamachado, elaine\} @icomp.ufam.edu.br
}

\begin{abstract}
Resumo. Uma disciplina de produção de textos passou a ser ministrada com suporte de um AVA que disponibiliza quizzes online como avaliação formativa. O objetivo deste artigo é identificar quais atributos do comportamento do estudante, registrados no AVA, têm maior associação com o seu desempenho. Por meio de uma análise exploratória, examinou-se a associação entre as notas parciais e os atributos coletados pelo AVA. Observou-se que a média de notas nos quizzes tem correlação positiva com as notas dos trabalhos de produção de texto. Esse fato deve ser considerado pelo instrutor ao elaborar os quizzes.
\end{abstract}

\begin{abstract}
A writing course was designed with the support of an LMS that provides online quizzes as formative assessment. The purpose of this article is to identify which attributes of student behavior, recorded in the LMS, have significant association with their performance. Using an exploratory analysis, we examined the association between the performance in the partial marks and the LMS attributes. We observed that the average quiz marks have a positive correlation with exam marks. This fact should be considered by instructors when elaborating online quizzes.
\end{abstract}

\section{Introdução}

No ensino superior, ambientes virtuais de aprendizagem (AVAs) têm sido cada vez mais usados para suplementar as aulas presenciais. Tal prática é uma das modalidades mais comuns de ensino híbrido [Schiehl and Gasparini 2017]. Como parte do curso híbrido é mediado por um sistema computacional, cada aprendiz deixa registrada uma sequência de ações, que podem incluir número de visitas a objetos de aprendizagem, duração e momento de início do acesso, notas recebidas, etc. A esse conjunto de informações chama-se comportamento do estudante [Sheshadri et al. 2018, Gholami and Zhang 2018].

O objetivo deste artigo é identificar quais atributos do comportamento do estudante, registrados no AVA, têm maior associação com o seu desempenho em uma disciplina de produção de textos. Para tanto, foram analisados os relatórios de um AVA utilizado em 2017 e 2018 como suporte à disciplina Textos Técnicos em Ciência da Computação (TTCC), ministrada para alunos do $1^{\circ}$ período do curso de Ciência da Computação da Universidade Federal do Amazonas (UFAM). Para nortear este estudo, levantaram-se as seguintes questões de pesquisa:

- Q1: Existe associação entre o desempenho do estudante no processo seletivo e seu desempenho em TTCC?

- Q2: Quais parâmetros dos quizzes online coletados pelo AVA têm maior associação com o desempenho do estudante em TTCC? 
VII Congresso Brasileiro de Informática na Educação (CBIE 2018)

Anais do XXIX Simpósio Brasileiro de Informática na Educação (SBIE 2018)

Este texto encontra-se organizado em seis seções. A Seção 2 apresenta alguns trabalhos relacionados ao comportamento de estudantes em quizzes online em disciplinas ministradas de forma híbrida. Na Seção 3, descreve-se o contexto da disciplina usada como estudo de caso. A Seção 4 descreve a amostra coletada e seus atributos, cuja análise é descrita na Seção 5. Por fim, as conclusões deste estudo são apresentadas na Seção 6.

\section{Revisão da Literatura}

Embora haja muitos relatos sobre comportamento de estudantes em Cursos Online Abertos e Massivos (MOOCs), pouco se conhece sobre a aplicabilidade desses resultados sobre cursos híbridos [Sheshadri et al. 2018]. Diferentemente dos MOOCs, nem todas as atividades de aprendizado do aluno podem ser registradas sistematicamente nos cursos híbridos. Por isso, os dados disponíveis podem não ser suficientes para realizar as mesmas análises e aplicar generalizações de uma modalidade de ensino para a outra [An et al. 2017].

Por exemplo, Gholami e Zhang (2018) estudaram o comportamento de estudantes em quizzes online não supervisionados na disciplina de Organização de Computadores, lecionada para cerca de 200 estudantes do $2^{\circ}$ ano de graduação, em uma classe híbrida. Os quizzes eram semanais, com prazo de 05 dias. O estudante tinha duas oportunidades de ver sua nota parcial antes do prazo e, com base nela, submeter uma nova tentativa, mas com penalidade sobre a nota final. Os autores notaram que os estudantes de melhor desempenho eram aqueles que iniciavam mais cedo a resolução dos quizzes, nunca perdiam os quizzes e mantinham um padrão estável de desempenho ao longo do curso. Também notaram que questões de desafio podem funcionar bem em avaliações presenciais, mas podem penalizar os estudantes de baixo desempenho em quizzes online.

De modo semelhante, Metz (2008) estudou o efeito do tempo de acesso aos quizzes sobre o desempenho de alunos em duas disciplinas da área de Biologia, ministradas em formato híbrido. Os quizzes também eram passados semanalmente, mas o tempo de acesso era menor: $72 \mathrm{~h}$ em um dos cursos e $24 \mathrm{~h}$ no outro. Uma vez iniciado o quiz, os alunos tinham apenas $20 \mathrm{~min}$ para completá-los. A autora também observou uma correlação negativa entre o tempo de antecedência no acesso ao quiz e o desempenho nas disciplinas. Especialmente, notou que os quizzes completados entre meia-noite e $8 \mathrm{~h}$ da manhã apresentaram pior desempenho do que quizzes completados em outras horas do dia.

Por fim, Sheshadri et al. (2018) sintetizaram dados de quatro plataformas de aprendizagem, utilizadas ao mesmo tempo em três turmas híbridas. Eles concluíram que o uso de vários sistemas adiciona complexidade para o estudante navegar entre elas, já que raramente duas plataformas eram usadas em uma mesma sessão de estudos. Além disso, as plataformas eram usadas alternadamente, em um comportamento dirigido por prazos, independentemente do desempenho do estudante.

Este trabalho alinha-se com os anteriores ao analisar o comportamento de estudantes em uma classe híbrida. Difere desses por manter um registro sistematizado das avaliações offline em forma de rubrica de correção. Além disso, preocupou-se em analisar a influência do conhecimento prévio do estudante sobre o desempenho da disciplina.

\section{A disciplina de Textos Técnicos em Ciência da Computação (TTCC)}

TTCC é ofertada para o $1^{\circ}$ período do curso de Ciência da Computação da UFAM. É uma disciplina de 30 horas, ministrada uma vez por semana em uma aula de 1 h50min 
VII Congresso Brasileiro de Informática na Educação (CBIE 2018)

Anais do XXIX Simpósio Brasileiro de Informática na Educação (SBIE 2018)

de duração. Seu objetivo é dotar o estudante com a habilidade de organizar e expressar ideias técnicas em um texto dissertativo, com clareza e coerência, segundo a norma culta da língua portuguesa.

Em 2013, TTCC foi ofertada pela primeira vez, na modalidade presencial. Em 2016, o AVA mantido pela instituição [Castro and Fuks 2009], baseado no Moodle, foi utilizado pela primeira vez para apoiar as atividades presenciais. Entre as atividades disponibilizadas, foram aplicados 11 quizzes como avaliação formativa. A maior parte das questões era focada em gramática e não cobria todo o assunto ministrado. Não havia questões de leitura e interpretação de textos. Quizzes podiam ser revisados indefinidamente até o prazo de submissão, o que gerou um comportamento predatório, por parte de alguns alunos, de marcar respostas aleatórias até obter nota 10. A partir de 2017, as submissões foram restritas a uma única tentativa e o material disponibilizado no AVA adquiriu maturidade, como se descreve a seguir.

\subsection{Os quizzes online}

A cada semana, o instrutor disponibiliza um quiz online para que os estudantes respondam até o início da aula seguinte. Os quizzes são constituídos por questões objetivas com correção automática: múltipla escolha de resposta única, múltipla escolha com mais de uma resposta correta, associação de conceitos, completamento de lacunas, entre outros.

Os quizzes são organizados em duas partes, de número variável de questões: interpretação de texto e o conteúdo que será abordado na aula seguinte. O objetivo da primeira parte é estimular os estudantes a lerem textos relacionados à computação, à ciência ou à educação, já que a produção de textos e o hábito de ler são habilidades estreitamente relacionadas [Cunha and Santos 2006]. O objetivo da segunda parte é estimular o estudo do conteúdo que será ministrado na aula seguinte.

As questões dos quizzes requerem um cuidado para serem elaboradas. Não basta coletar questões disponíveis em gramáticas e manuais de concursos. Como princípio básico, nenhuma questão deve se limitar a pedir a classificação de uma palavra ou oração de acordo com a morfologia ou sintaxe, pois o objetivo da disciplina é desenvolver no estudante a habilidade de produzir bons textos, independentemente de seu conhecimento sobre classificar corretamente palavras e orações. Além disso, os exemplos utilizados nas questões devem ser colhidos de textos sobre computação, ciência ou vida acadêmica. Por fim, os próprios textos produzidos pelos alunos são utilizados como base para elaborar questões sobre falhas na escrita.

Os quizzes ficam disponíveis por uma semana, acessíveis a qualquer momento e local. As respostas podem ser alteradas durante o prazo de submissão. Ao fim deste, as tentativas de resposta são enviadas automaticamente, recurso útil para uma turma composta de calouros, com pouca familiaridade com o AVA. O feedback das questões é fornecido somente após o prazo de entrega.

\subsection{As avaliações}

Desde 2017, são passados 13 quizzes no AVA. A média dos quizzes vale 16,7\% da nota final na disciplina. As demais avaliações são compostas por três trabalhos parciais, de caráter sumativo, com peso de 16,7\% cada, e um trabalho final, com peso de $33,3 \%$. 
VII Congresso Brasileiro de Informática na Educação (CBIE 2018)

Anais do XXIX Simpósio Brasileiro de Informática na Educação (SBIE 2018)

$\mathrm{O} 1^{\circ}$ e o $3^{\circ}$ trabalho parcial, bem como o final, requerem que o estudante elabore um texto dissertativo-argumentativo sobre um tema relacionado à computação ou à vida acadêmica. Os temas são mudados a cada ano, mas pelo menos um dos três trabalhos aborda o tema ética. O Trabalho 2 é desenvolvido em grupos de dois ou três alunos e envolve a reescrita de textos.

Para corrigir os trabalhos de produção de textos, adota-se uma rubrica composta de 21 itens, distribuídos em quatro eixos: Organização, Conteúdo, Expressão escrita e Mecânica do texto. A rubrica é divulgada na especificação dos trabalhos e gera uma nota de 0 a 10, que não é a nota definitiva do trabalho. Isso permite que resultados de diferentes trabalhos e períodos possam ser comparados. A nota final resulta de bonificações ou penalidades aplicadas sobre a nota obtida a partir da rubrica, conforme requisitos listados na especificação, que guardam relação com o assunto ministrado à época da atividade. $\mathrm{O}$ texto submetido pelo aluno é avaliado com respeito a cada um dos itens da rubrica em uma escala Likert que varia de 1 a 4 . A largura da escala é propositadamente par, a fim de forçar que o avaliador reflita se o texto do aluno atende bem ou não um determinado item da rubrica e não recaia na comodidade de pontuar um valor intermediário.

Os alunos submetem os arquivos de texto pelo mesmo AVA onde são disponibilizados os quizzes. O professor imprime todos os textos e os avalia, destacando os problemas encontrados. A nota é indicada apenas na planilha, para que o aluno a consulte e verifique quais itens da rubrica precisa aprimorar.

O formato dos trabalhos deve seguir obrigatoriamente o modelo de artigos da SBC, estimulando-se, desse modo, o aluno a manusear um formato de texto de divulgação científica. Os textos também devem ser produzidos em um editor L $\mathrm{HT}_{\mathrm{E}} \mathrm{X}$. Dessa forma, o estudante é estimulado a adequar-se rapidamente a uma mudança tecnológica, competência preconizada pelas Diretrizes Curriculares Nacionais para os cursos de graduação em Computação [MEC 2016].

\section{Método}

O presente estudo foi delineado na forma de um projeto de pesquisa-ação, em que dois ciclos foram completados e o terceiro está próximo de se encerrar. Esse delineamento combina vários estágios, que incluem a identificação de um problema (como melhorar a qualidade dos textos produzidos?), a abordagem do problema (introduzir quizzes online), avaliação e reflexão da intervenção, e posterior modificação da prática.

\subsection{Caracterização das Amostras}

Este estudo considerou dados de 2014 a 2018. De 2014 a 2016, analisaram-se apenas as notas nos processos seletivos e nos trabalhos práticos. A partir de 2017, passou-se a considerar também os dados dos quizzes online. Foram considerados apenas os estudantes que ingressaram no ano em que a disciplina foi lecionada, cujo ingresso tenha sido realizado pelo PSC ou pelo SiSU (vide Seção 4.2), e que não tenham desistido formalmente do curso ou abandonado a disciplina. A Tabela 1 apresenta, para cada ano, as seguintes informações: número de alunos matriculados; entre estes, o número de alunos frequentantes das aulas presenciais; e entre estes, o número de alunos que resolveram pelo menos a metade dos quizzes. Para os alunos frequentantes, também são apresentadas a proporção de mulheres e homens, e a idade média no primeiro dia de aula, com o desvio padrão. 
VII Congresso Brasileiro de Informática na Educação (CBIE 2018)

Anais do XXIX Simpósio Brasileiro de Informática na Educação (SBIE 2018)

Tabela 1. Caracterização das amostras

\begin{tabular}{rrrrrrr}
\hline Ano & \#Alunos Matr. & \#Alunos Freq. & \#Alunos Quiz & \% Mulheres & \% Homens & Idade \\
\hline 2014 & 54 & 49 & - & $24,5 \%$ & $75,5 \%$ & $19,5 \pm 2,7$ \\
2015 & 61 & 58 & - & $12,1 \%$ & $87,9 \%$ & $19,4 \pm 2,0$ \\
2016 & 54 & 54 & - & $13,0 \%$ & $87,0 \%$ & $19,0 \pm 0,9$ \\
2017 & 58 & 48 & 48 & $25,0 \%$ & $75,0 \%$ & $19,4 \pm 2,5$ \\
2018 & 63 & 49 & 46 & $24,5 \%$ & $75,5 \%$ & $19,5 \pm 2,2$ \\
\hline
\end{tabular}

\subsection{Dados coletados}

Para conduzir a análise, foram coletados os seguintes dados:

- TrabX-P: nota parcial do trabalho X (1 ou 3), proveniente da rubrica de correção.

- TrabX-F: nota final do trabalho X (1 ou 3), registrada em boletim, calculada a partir da aplicação de bonificações e penalidades sobre a nota parcial.

- Média quizzes: média das notas obtidas nos quizzes disponibilizados no AVA.

- \# Quizzes: quantidade de quizzes resolvidos pelo estudante no AVA.

- Antecedência: tempo de antecedência (em h) ao prazo com que os estudantes iniciavam a resolução dos quizzes.

- \# Faltas: quantidade de faltas do estudante às aulas presenciais.

- Enem: conjunto de cinco notas (0 a 1000) obtidas pelos estudantes nas provas do Enem $^{1}$ : Linguagens e Códigos (L), Ciências da Natureza (CN), Ciências Humanas $(\mathrm{CH})$, Matemática (M) e Redação (R), utilizadas para ingressar em $50 \%$ das vagas, ofertadas pelo Sistema de Seleção Unificada (SiSU). Quando disponível, foram consideradas as notas do Enem dos estudantes que ingressaram via PSC.

- PSC: nota geral obtida pelo estudante no Processo Seletivo Contínuo ${ }^{2}$ (PSC), aplicado durante os três anos do Ensino Médio aos estudantes do estado do Amazonas. Essa nota (0 a 500) é utilizada pelo estudante para ingressar nas demais vagas. Não foi possível obter acesso à nota individualizada por prova, por isso utilizou-se a nota consolidada. Quando disponível, foram consideradas as notas do PSC dos estudantes que ingressaram via SiSU.

Tais dados foram coletados a partir das seguintes fontes:

- Relatórios de notas de cada quiz realizado, fornecidos pelo AVA.

- Planilha de notas do instrutor, contendo as notas dos alunos em cada trabalho.

- Planilha de notas dos candidatos optantes pelo SiSU.

- Planilha pública de classificação dos candidatos ao PSC.

\section{Análise e Resultados}

Nesta seção, apresentam-se os resultados do presente estudo. Primeiro, verificou-se a associação entre desempenho nos processos seletivos e desempenho na disciplina, analisando-se dados de 2014 a 2018. Em seguida, focou-se nos ciclos de 2017 e 2018 para analisar os atributos colhidos nos relatórios de notas do AVA, com a finalidade de caracterizar o comportamento do estudante e entender seu reflexo no desempenho da disciplina. Os testes estatísticos foram realizados no software $R$ versão 3.5.0. O nível de significância utilizado foi $\alpha<0,05$.

\footnotetext{
${ }^{1}$ https://enem.inep.gov.br/

${ }^{2}$ http: //www. comvest.ufam.edu.br/psc.htm
} 
VII Congresso Brasileiro de Informática na Educação (CBIE 2018)

Anais do XXIX Simpósio Brasileiro de Informática na Educação (SBIE 2018)

\subsection{Associação entre desempenho nos processos seletivos e desempenho em TTCC}

Como o foco da disciplina TTCC é a produção de textos, o senso comum diz que o desempenho nela estaria relacionado ao desempenho do estudante nas provas de seleção, em especial a prova de Redação.

Para verificar essa conjectura de forma objetiva, é necessário explicitar primeiramente qual a medida de "desempenho na disciplina". Pode-se tomar a média final do aluno (MF), mas esse fator é influenciado pelo conteúdo da disciplina, bem como pela metodologia de ensino-aprendizagem. Outra opção é tomar a nota final do Trabalho 1, a primeira avaliação realizada na disciplina. Uma terceira opção é tomar a nota parcial do Trabalho 1, à qual não foram aplicadas penalidades e bonificações relacionadas ao assunto abordado. De forma exploratória, verificou-se a associação dessas três medidas com respeito às notas do Enem e PSC.

A Tabela 2 exibe quantos alunos de Ciência da Computação participaram de cada processo seletivo de 2014 a 2018. Não se verificou o ano de 2013, primeiro ano da aplicação da disciplina, porque não se adotava a rubrica para avaliar os trabalhos.

Tabela 2. Participação dos ingressantes nos processos seletivos de 2014 a 2018

\begin{tabular}{rrrrrr}
\hline & \multicolumn{5}{c}{ Ano } \\
\cline { 2 - 6 } Processo seletivo & $\mathbf{2 0 1 4}$ & $\mathbf{2 0 1 5}$ & $\mathbf{2 0 1 6}$ & $\mathbf{2 0 1 7}$ & $\mathbf{2 0 1 8}$ \\
\hline Enem & 31 & 38 & 51 & 29 & 37 \\
PSC & 36 & 35 & 41 & 32 & 30 \\
Enem + PSC & 18 & 15 & 38 & 13 & 18 \\
\hline Total & 49 & 58 & 54 & 48 & 49 \\
\hline
\end{tabular}

Antes de testar a correlação, verificou-se a normalidade da distribuição das grandezas envolvidas, por meio teste de Shapiro-Wilk: as notas das cinco provas do Enem, a nota geral no Enem, a nota geral do PSC, a média final em TTCC, e as notas final e parcial do Trabalho 1. Todas as notas obtidas pelos alunos nos dois processos seletivos de 2014 a 2018 apresentaram distribuição normal $(p>0,05)$. Porém, nenhuma das três medidas de desempenho em TTCC apresentaram distribuição normal.

Por isso, aplicou-se a correlação não-paramétrica de Kendall para medir o grau de associação entre o conhecimento prévio dos alunos, expresso pelas notas dos processos seletivos, e cada uma das três medidas de desempenho em TTCC.

A Tabela 3 apresenta o resultado dessas correlações apenas para a nota parcial do Trabalho 1. Quando a nota final no Trabalho 1 e média final na disciplina foram tomadas como medida de desempenho, foram observadas uma menor quantidade de correlações significativas (valor $p<0,05$ ). A partir da Tabela 3, observa-se que, de 2014 a 2016, havia uma correlação entre as notas nos processos seletivos e a nota parcial no Trabalho 1 , mas deixou de haver em 2017 e 2018, exceto pela prova de Redação (R) do Enem em 2018 $(\tau=0,33, p=0,0046)$. As provas que mais apresentaram correlações significativas, em três dos cinco anos analisados, foram Linguagens e Códigos (L), Ciências Naturais (CN) e Matemática (M), e a nota geral do Enem. Verifica-se ainda que a correlação vai caindo de intensidade entre 2014 e 2016, deixando de ser significativa em 2017 e 2018. 
VII Congresso Brasileiro de Informática na Educação (CBIE 2018)

Anais do XXIX Simpósio Brasileiro de Informática na Educação (SBIE 2018)

Tabela 3. Correlação entre notas nos processos seletivos e nota parcial do Trabalho 1. As correlações significativas (valor $p<0,05$ ) estão marcadas em negrito

\begin{tabular}{|c|c|c|c|c|c|c|c|c|c|c|}
\hline & \multicolumn{2}{|c|}{2014} & \multicolumn{2}{|c|}{2015} & \multicolumn{2}{|c|}{2016} & \multicolumn{2}{|c|}{2017} & \multicolumn{2}{|c|}{2018} \\
\hline & $\tau$ & $p$ & $\tau$ & $p$ & $\tau$ & $p$ & $\tau$ & $p$ & $\tau$ & $p$ \\
\hline PSC & 0,54 & $\mathbf{0 , 0 0 0 0}$ & 0,36 & $\mathbf{0 , 0 1 1 1}$ & $-0,08$ & 0,5249 & 0,20 & 0,1116 & 0,24 & 0,0683 \\
\hline Enem-L & 0,49 & 0,0011 & 0,42 & $\mathbf{0 , 0 0 2 7}$ & 0,36 & 0,0015 & 0,16 & 0,2397 & 0,16 & 0,1811 \\
\hline Enem-CH & 0,49 & 0,0011 & 0,12 & 0,3898 & 0,37 & 0,0012 & 0,12 & 0,3613 & $-0,07$ & 0,5206 \\
\hline Enem-CN & 0,66 & 0,0000 & 0,28 & 0,0472 & 0,23 & 0,0442 & 0,05 & 0,7239 & 0,04 & 0,7431 \\
\hline Enem-M & $\mathbf{0 , 4 1}$ & 0,0069 & 0,41 & 0,0031 & 0,24 & 0,0325 & 0,04 & 0,7520 & 0,06 & 0,6276 \\
\hline Enem-R & 0,36 & 0,0219 & 0,20 & 0,1568 & 0,08 & 0,5110 & 0,11 & 0,4063 & $\mathbf{0 , 3 3}$ & 0,0046 \\
\hline EnemTotal & 0,56 & 0,0002 & 0,43 & 0,0023 & $\mathbf{0 , 3 0}$ & 0,0089 & 0,12 & 0,3813 & 0,16 & 0,1686 \\
\hline
\end{tabular}

Por fim, verifica-se que a prova de redação teve correlação com o desempenho em TTCC somente em 2014 e 2018. Dessa forma, para efeitos do estudo do comportamento dos estudantes no AVA, adotado em 2017 e 2018, verifica-se que o desempenho em TTCC não possui correlação linear com o desempenho nos exames de seleção. Entendida essa falta de associação, foram analisados os relatórios dos quizzes, a fim de verificar associação entre o comportamento do estudante no AVA e seu desempenho na disciplina.

\subsection{Associação entre parâmetros do quiz e desempenho na disciplina}

Para responder à segunda questão de pesquisa, as seguintes variáveis foram usadas como parâmetros dos quizzes: quantidade média de quizzes resolvidos (\# Quizzes), nota média obtida nos quizzes (Média Quizzes) e tempo de antecedência de início dos quizzes (Antecedência). Como nenhuma delas apresenta distribuição normal (teste de Shapiro-Wilk), usou-se a correlação de Kendall para testar sua associação com as notas dos trabalhos.

Como medida de desempenho individual, consideraram-se as notas parciais e finais dos Trabalhos 1 e 3, em 2017 e 2018. O Trabalho 2 não foi analisado por ser resolvido em grupo. A média final também não foi usada porque 16,7\% de seu valor é determinado pela média dos quizzes. Por fim, não foi possível analisar as notas parciais do Trabalho 3 (Trab3-P) de 2017, pois o arquivo que as continha foi corrompido. Nos testes de correlação entre médias dos quizzes e notas de trabalhos, consideraram-se apenas os quizzes realizados antes da entrega do trabalho analisado: 5 antes do Trabalho 1 em 2017, 6 antes do Trabalho 1 em 2018, 12 antes do Trabalho 3 em 2017 e 2018.

Tabela 4. Correlação entre parâmetros do quiz e desempenho nos trabalhos de 2017 e 2018. As correlações significativas $(p<0,05)$ estão marcadas em negrito

\begin{tabular}{|c|c|c|c|c|c|c|c|c|c|c|c|c|c|c|}
\hline & \multicolumn{6}{|c|}{2017} & \multicolumn{8}{|c|}{2018} \\
\hline & \multicolumn{2}{|c|}{ Trab1-F } & \multicolumn{2}{|c|}{ Trab1-P } & \multicolumn{2}{|c|}{ Trab3-F } & \multicolumn{2}{|c|}{ Trab1-F } & \multicolumn{2}{|c|}{ Trab1-P } & \multicolumn{2}{|c|}{ Trab3-F } & \multicolumn{2}{|c|}{ Trab3-P } \\
\hline & $\tau$ & $p$ & $\tau$ & $p$ & $\tau$ & $p$ & $\tau$ & $p$ & $\tau$ & $p$ & $\tau$ & $p$ & $\tau$ & $p$ \\
\hline \# Quiz & 0,05 & 0,66 & 0,03 & 0,80 & 0,24 & $\mathbf{0 , 0 3}$ & 0,22 & 0,07 & 0,23 & 0,06 & 0,29 & 0,02 & 0,28 & 0,02 \\
\hline Média Quiz & 0,21 & 0,04 & 0,15 & 0,14 & 0,27 & 0,01 & 0,31 & 0,00 & 0,29 & 0,00 & 0,35 & 0,00 & 0,30 & 0,01 \\
\hline Antecedência & $-0,09$ & 0,39 & $-0,07$ & 0,49 & 0,09 & 0,38 & 0,20 & 0,07 & 0,26 & 0,01 & $-0,04$ & 0,69 & $-0,03$ & 0,76 \\
\hline
\end{tabular}


VII Congresso Brasileiro de Informática na Educação (CBIE 2018)

Anais do XXIX Simpósio Brasileiro de Informática na Educação (SBIE 2018)

A Tabela 4 apresenta o resultado dos testes de correlação. Verifica-se que a média dos quizzes é o parâmetro que guarda correlação significativa (ou seja, diferente de zero) com mais indicadores de desempenho em TTCC: seis das sete notas de trabalhos. Embora significantes, as correlações são fracas $(<0,40)$, semelhantes às encontradas por [Sheshadri et al. 2018] e [Gholami and Zhang 2018]. A multiplicidade de fatores em um contexto educacional pode explicar esses baixos valores. Além disso, as maiores correlações encontradas foram com a nota final do Trabalho 3 (Trab3-F): $\tau=0,27 \mathrm{em} 2017 \mathrm{e}$ $\tau=0,35 \mathrm{em} 2018$. Isso pode ser um indício de que o contato semanal com os quizzes faz com que o estudante cometa menos erros de escrita.

Para entender melhor como a nota final do Trabalho 3 se relaciona com os demais parâmetros dos quizzes, os estudantes foram agrupados em quatro categorias de nota: entre 9 e 10, entre 7 e 9, entre 5 e 7, e abaixo da média de aprovação (5,0). As Tabelas 5 e 6 apresentam os resultados para os anos de 2017 e 2018, respectivamente. Observa-se que os alunos com o pior desempenho no Trabalho 3 têm, em geral, as piores médias nos quizzes (o que foi medido anteriormente pelo teste de correlação), o menor número de quizzes resolvidos e as maiores quantidades de faltas às aulas presenciais. Dessa forma, verifica-se que o desempenho em TTCC está associado à presença às atividades em sala de aula, bem como às atividades do AVA, em especial, os quizzes online. Portanto, o quiz é um recurso que deve ser aprimorado a cada nova oferta da disciplina.

Tabela 5. Medidas de desempenho por categoria do Trab3-F em 2017

\begin{tabular}{rrrrrr}
\hline Categoria & \# Alunos & Nota no quiz & \# Quizzes & Antecedência (h) & \# Faltas \\
\hline 9 a 10 & 5 & 8,18 & 10,60 & 48,08 & 3,8 \\
7 a 8,99 & 6 & 9,29 & 12,00 & 54,35 & 0,8 \\
5 a 6,99 & 13 & 8,82 & 11,62 & 74,03 & 1,2 \\
0 a 4,99 & 24 & 7,83 & 10,92 & 51,01 & 2,8 \\
\hline Total & 48 & 8,32 & 11,21 & 57,36 & 2,2 \\
\hline
\end{tabular}

Tabela 6. Medidas de desempenho por categoria do Trab3-F em 2018

\begin{tabular}{rrrrrr}
\hline Categoria & \# Alunos & Nota no quiz & \# Quizzes & Antecedência (h) & \# Faltas \\
\hline 9 a 10 & 7 & 7,73 & 11,43 & 26,56 & 4,3 \\
7 a 8,99 & 6 & 7,33 & 11,33 & 27,14 & 2,7 \\
5 a 6,99 & 3 & 7,75 & 12,00 & 16,99 & 0,0 \\
0 a 4,99 & 30 & 6,35 & 10,67 & 29,97 & 4,6 \\
\hline Total & 46 & 6,78 & 10,96 & 23,23 & 4,0 \\
\hline
\end{tabular}

\section{Procrastinação e desempenho nas avaliações}

Os testes de correlação e os dados das Tabelas 5 e 6 mostram a ausência de associação entre o tempo de antecedência de resolução dos quizzes e o desempenho em TTCC em 2017 e em 2018. Por outro lado, a mediana do tempo de início de resolução dos quizzes caiu de 51,3h antes do prazo em 2017 para 19,3h em 2018. Da mesma maneira, observou-se que 
VII Congresso Brasileiro de Informática na Educação (CBIE 2018)

Anais do XXIX Simpósio Brasileiro de Informática na Educação (SBIE 2018)

2017

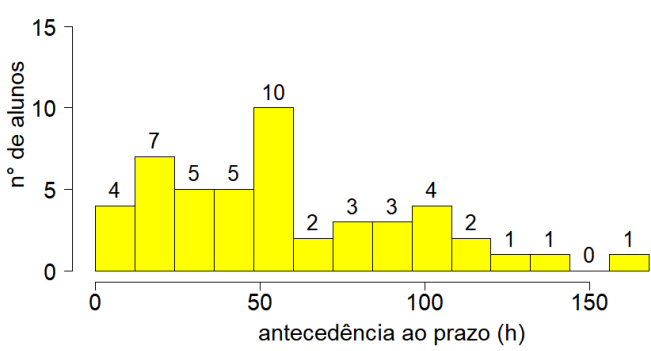

2018

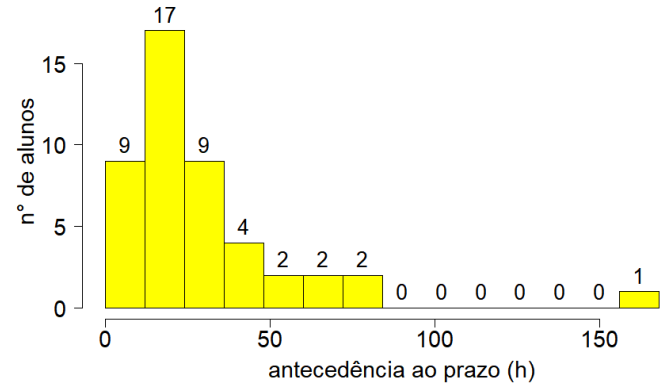

Figura 1. Distribuição do tempo antecedência de resolução dos quizzes

a mediana da nota do Trab3-F caiu de 5,0 para 0,0. Essa mudança de hábito de acesso aos quizzes de 2017 para 2018 pode ser mais bem observada nos histogramas da Figura 1. Neles, cada barra representa um período de $12 \mathrm{~h}$. Veem-se também valores atípicos, acima de $168 \mathrm{~h}$ (uma semana), o que é explicado por feriados, que ocasionalmente distanciam aulas consecutivas em duas semanas.

Verifica-se que, em 2017, 27 alunos iniciavam os quizzes com pelo menos 48h de antecedência. É um tempo adequado para ler as questões, pesquisar a resposta, discutilas com colegas, revisar e submeter. Já em 2018, apenas 7 alunos apresentaram esse comportamento. Não por acaso, o teste de amostras não pareadas de Wilcoxon mostrou que houve diferença significativa nas notas finais do Trabalho 1 (Trab1-F) entre as turmas de 2017 e 2018 ( $p \approx 0,0021$ ). Da mesma forma, tomando-se as notas finais do Trabalho 3 (Trab3-F), também se verificou diferença significativa no desempenho entre os dois anos ( $p \approx 0,0270$ ). Os boxplots da Figura 2 mostram graficamente essa diferença.

O aumento na variação de notas entre o Trab1-F e Trab3-F se deve a dois movimentos opostos: alunos que se desdobram para recuperar a nota e outros que desistem e deixam de entregar o Trabalho 3. Estes continuam indo às aulas até o limite de faltas e resolvendo os quizzes, mas não realizam a tarefa mais complexa, a produção de texto.
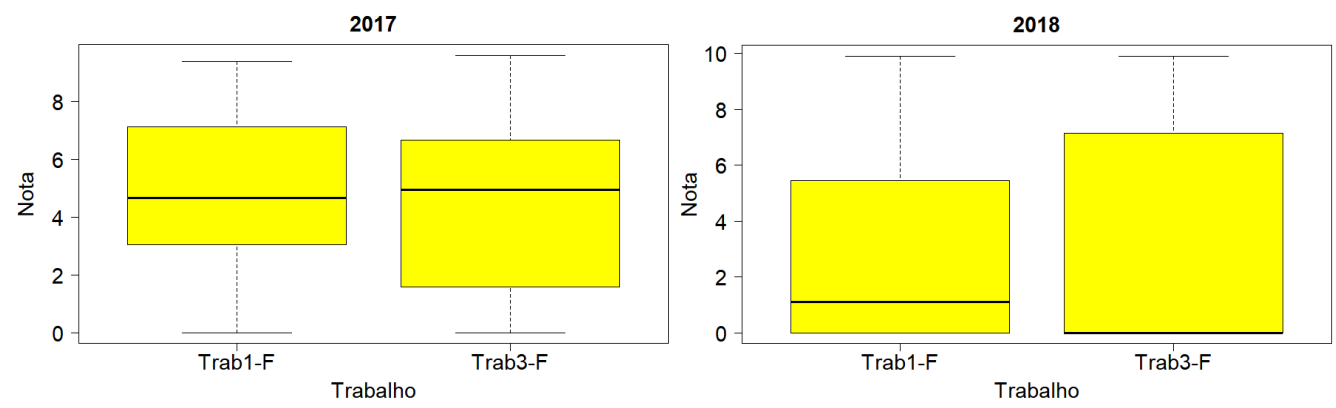

Figura 2. Distribuição das notas finais dos Trabalhos 1 e 3

\section{Conclusão}

Um AVA foi adotado como ferramenta de apoio a uma disciplina de produção de textos técnicos. Quizzes online com correção automática passaram a ser disponibilizados semanalmente para melhorar o desempenho nas atividades produção de textos. Verificou-se que em 2014 havia correlação positiva moderada $(0,4<\tau<0,7)$ entre as notas do 
VII Congresso Brasileiro de Informática na Educação (CBIE 2018)

Anais do XXIX Simpósio Brasileiro de Informática na Educação (SBIE 2018)

Trabalho 1 e as notas nas provas dos processos seletivos (Enem e PSC). Nos dois anos seguintes, o grau de correlação caiu para positiva fraca $(\tau<0,4)$. A partir de 2017, a correlação deixou de ser significativa. De forma independente, também em 2017, os quizzes passaram a ser adotados como avaliação formativa.

Nos dois anos de uso (2017 e 2018), verificou-se que a nota média nos quizzes é um atributo associado a um bom desempenho nos trabalhos de produção de textos, usados como avaliação somativa, corroborando com estudos prévios [Gholami and Zhang 2018, Sheshadri et al. 2018]. Diferente de [Gholami and Zhang 2018], não se encontrou correlação entre desempenho e quantidade de quizzes resolvidos, o que possivelmente se credita ao pequeno tamanho da população estudada $(\approx 50$ por ano, em contraste a $\approx 200)$. Por outro lado, observou-se que estudantes com notas abaixo do limiar de aprovação apresentaram as menores quantidades de quizzes resolvidos e o maior número de faltas às aulas presenciais. Além disso, verificou-se diferença de comportamento entre as turmas de 2017 e 2018 com respeito ao tempo de antecedência de resolução dos quizzes, o que provavelmente explica a diferença de desempenho encontrada.

Como trabalho futuro, pretende-se considerar outros atributos do AVA, a fim de investigar um modelo de predição. Também é preciso realizar análises qualitativas, como, por exemplo, verificar a sensação de aprendizado do estudante com cada quiz.

\section{Agradecimentos}

Agradecemos à Yara Renovato (PROEG/UFAM) pelo acesso aos dados do Sistema de Informações para o Ensino (SIE/UFAM).

\section{Referências}

An, T.-S., Krauss, C., and Merceron, A. (2017). Can Typical Behaviors Identified in MOOCs be Discovered in Other Courses? In Proceedings of The 10th International Conference on Educational Data Mining (EDM 2017), pages 25-28.

Castro, T. and Fuks, H. (2009). Inspeção Semiótica do ColabWeb: Proposta de Adaptações para o Contexto da Aprendizagem de Programação. RBIE, 17(01):71-81.

Cunha, N. B. and Santos, A. A. A. (2006). Relação entre a compreensão da leitura e a produção escrita em universitários. Psicologia: Reflexão e Crítica, 19:237-245.

Gholami, A. and Zhang, L. Y. (2018). Student Behaviour in Unsupervised Online Quizzes: A Closer Look. In Proceedings of the 23rd Western Canadian Conference on Computing Education, WCCCE'18, pages 8:1-8:6. ACM.

MEC (2016). Diretrizes Curriculares Nacionais para os Cursos de Graduação em Computação. Disponível em https : / / goo.gl/35 CmzT (Acesso em jun. 2018).

Metz, A. M. (2008). The effect of access time on online quiz performance in large biology lecture courses. Biochemistry and Molecular Biology Education, 36(3):196-202.

Schiehl, E. P. and Gasparini, I. (2017). Modelos de ensino híbrido: Um mapeamento sistemático da literatura. In Simpósio Brasileiro de Informática na Educação (SBIE).

Sheshadri, A., Gitinabard, N., F Lynch, C., Barnes, T., and Heckman, S. (2018). Predicting Student Performance Based on Online Study Habits: A Study of Blended Courses. In Educational Data Mining. 